PROCEEDINGS OF THE

AMERICAN MATHEMATICAL SOCIETY

Volume 125, Number 11, November 1997, Pages 3155-3161

S 0002-9939(97)04049-5

\title{
THE $Q$-SPECTRUM AND SPANNING TREES OF TENSOR PRODUCTS OF BIPARTITE GRAPHS
}

\author{
TIMOTHY Y. CHOW \\ (Communicated by Jeffry N. Kahn)
}

\begin{abstract}
Recently, Knuth and Ciucu independently proved the surprising fact, conjectured by Stanley, that one connected component of the tensor product of a path with itself (the so-called "Aztec diamond graph") has four times as many spanning trees as the other connected component, independent of the length of the path. We show here that much more is true: the connected components of the tensor product of any connected bipartite multigraphs all have essentially the same $Q$-spectrum. It follows at once that there is a simple formula relating their numbers of spanning trees.
\end{abstract}

\section{Introduction}

A spanning tree of a finite undirected graph is a connected acyclic subgraph with the same vertex set as the graph itself. The complexity of a graph is the number of spanning trees it has. The tensor product $G_{1} \otimes G_{2}$ of two graphs $G_{1}$ and $G_{2}$ is the graph whose adjacency matrix is the tensor product of the adjacency matrices of $G_{1}$ and $G_{2}$. In 1994, Stanley [13] conjectured that if $P_{2 n+1}$ is the path with $2 n+1$ vertices, then the complexity of one connected component of $P_{2 n+1} \otimes P_{2 n+1}$ (the Aztec diamond graph $\mathrm{AD}_{n}$, whose perfect matchings are studied in [4]) is four times the complexity of the other connected component (the odd Aztec diamond graph $\left.\mathrm{OD}_{n}\right)$. As an indication of why this conjecture is unexpected, we mention that the Laplacian spectra $([2], \S 1.2,(1.16))$ of $\mathrm{AD}_{n}$ and $\mathrm{OD}_{n}$ are quite different from each other. Also, virtually all known results about complexities of tensor products require some regularity assumption (e.g., [5], Theorem 6.10), but paths are not regular.

Recently, the conjecture was independently proved by Knuth [8] and Ciucu [1]. Knuth uses an idea of Cvetković and Gutman [3], exploiting the fact that $\mathrm{AD}_{n}$ and $\mathrm{OD}_{n}$ are nearly regular and are nearly planar duals of each other to reduce the problem to a question about the ordinary spectrum (i.e., the eigenvalues of the adjacency matrix), which behaves well under tensor product. Ciucu uses a bijection of Temperley [14] to transform the problem into a question about perfect matchings, and then derives Stanley's conjecture as a corollary of a matching theorem.

Received by the editors May 16, 1996.

1991 Mathematics Subject Classification. Primary 05C50, 05C05, 05C30; Secondary 15A18, $15 \mathrm{~A} 69$.

Key words and phrases. $Q$-spectrum, Laplacian, spanning tree enumeration, matrix-tree theorem, Aztec diamond, Prüfer codes.

The author was supported in part by an NSF postdoctoral fellowship. 
While both methods solve the original problem elegantly, they leave many questions unanswered. For example, how far can Stanley's conjecture be generalized? Is planarity, which is used in an essential way by both Knuth and Ciucu, essential to the phenomenon?

In this paper we show that the result generalizes to the tensor product of arbitrary connected bipartite multigraphs. (In particular, planarity is not needed.) The key observation, which is simple but does not seem to have been made before, is that the so-called $Q$-spectrum ([2], $\S 1.2,(1.6))$, which is known to essentially determine the complexity $([2], \S 1.9, \# 10)$, behaves well under tensor product. The main tool we use is a little-known result of Runge ([2], §1.9, \#11) on the $Q$-spectra of bipartite multigraphs.

\section{Preliminaries}

It will be convenient to make a few technical modifications to the definitions given in the introduction. For our purposes, a graph is a finite undirected graph without loops or multiple edges and with a weight associated to each edge. The degree $\operatorname{deg}(v)$ of a vertex $v$ is the sum of the weights of the edges incident to $v$. The adjacency matrix of a graph with vertex set $V=\left\{v_{1}, v_{2}, \ldots, v_{n}\right\}$ is the matrix whose $(i, j)$ th entry is the weight of the edge connecting $v_{i}$ and $v_{j}$ if such an edge exists, and whose $(i, j)$ th entry is zero otherwise. If $A=\left(a_{i j}\right)$ is an $m \times n$ matrix and $A^{\prime}=\left(a_{i j}^{\prime}\right)$ is an $m^{\prime} \times n^{\prime}$ matrix, then the tensor product $A \otimes A^{\prime}$ is the $m m^{\prime} \times n n^{\prime}$ matrix obtained by replacing the element $a_{i j}$ in $A$ by the matrix $a_{i j} A^{\prime}$. The tensor product $G_{1} \otimes G_{2}$ of two graphs $G_{1}$ and $G_{2}$ is the graph whose adjacency matrix is the tensor product of the adjacency matrices of $G_{1}$ and $G_{2}$. Note that the tensor product is associative.

A spanning tree of a graph $G$ is a connected acyclic subgraph of $G$ with the same vertex set as $G$. The weight $\operatorname{wt}(T)$ of a spanning tree $T$ is the product of the weights of its edges. The complexity $t(G)$ of a graph $G$ is defined by

$$
t(G) \stackrel{\text { def }}{=} \sum_{T \subset G} \mathrm{wt}(T)
$$

where the sum is over all spanning trees of $G$.

We have already mentioned that the key concept in our proof is the $Q$-spectrum, which we shall now define. More precisely, we shall define the $\phi$-polynomial of a bipartite graph, which is equivalent to its $Q$-spectrum (see [2], §1.9, \#11; or [11]).

First, given a matrix $A=\left(a_{i j}\right)$ whose row-sums $\sum_{j} a_{i j}$ are nonzero, define its stochasticization $S(A)$ to be the matrix obtained from $A$ by dividing each entry by the row-sum of the row it is in. Next, let $G$ be a connected bipartite graph with positive integer weights on all its edges and with at least one edge. Let $(X, Y)$ be a bipartition of $G$. If we order the vertices of $G$ so that all the $X$ vertices precede all the $Y$ vertices, then the adjacency matrix of $G$ looks like

$$
\left(\begin{array}{cc}
0 & A \\
A^{T} & 0
\end{array}\right) \text {. }
$$

We define the $\phi$-polynomial of $G$ by

$$
\phi_{G}(\lambda) \stackrel{\operatorname{def}}{=} \begin{cases}\operatorname{det}\left[\lambda I-S(A) S\left(A^{T}\right)\right], & \text { if }|X| \leq|Y| \\ \operatorname{det}\left[\lambda I-S\left(A^{T}\right) S(A)\right], & \text { if }|X|>|Y| .\end{cases}
$$


(Here $I$ is the identity matrix.) Note that since $G$ is connected, the bipartition $(X, Y)$ is essentially unique, so $\phi_{G}$ is uniquely determined by $G$.

We can now state the theorem of Runge.

Lemma 1. Let $G$ be a connected bipartite graph with positive integer weights on all its edges and at least one edge. Then $\lambda=1$ is a root of $\phi_{G}(\lambda)$, and if the roots of $\phi_{G}(\lambda)$ are $\lambda_{1}=1, \lambda_{2}, \ldots, \lambda_{k}$ and the sum of the weights of the edges of $G$ is $m$, then

$$
t(G)=\frac{\prod_{v \in G} \operatorname{deg}(v)}{m} \prod_{i=2}^{k}\left(1-\lambda_{i}\right) .
$$

Proof. We refer the reader to [11]. The proof there is given only for unit edge weights, but it generalizes immediately to arbitrary positive integer weights.

We need two other lemmas. The first of these is easy to prove, but in a sense it is the heart of the whole proof, because it shows the relationship between tensor products and the stochasticization operator.

Lemma 2. Let $A_{1}, A_{2}, \ldots, A_{n}$ be matrices whose row-sums are nonzero. Then the row-sums of $A_{1} \otimes A_{2} \otimes \cdots \otimes A_{n}$ are nonzero, and

$$
S\left(A_{1} \otimes A_{2} \otimes \cdots \otimes A_{n}\right)=S\left(A_{1}\right) \otimes S\left(A_{2}\right) \otimes \cdots \otimes S\left(A_{n}\right) .
$$

Proof. By induction it suffices to consider the case $n=2$. It is clear from the definitions that each row-sum of $A_{1} \otimes A_{2}$ is a row-sum of $A_{1}$ times a row-sum of $A_{2}$. Each element of $S\left(A_{1} \otimes A_{2}\right)$ is obtained by multiplying an element $a_{1}$ of $A_{1}$ by an element $a_{2}$ of $A_{2}$, and then dividing by a row-sum of $A_{1} \otimes A_{2}$. This is the same as dividing $a_{1}$ by the appropriate row-sum of $A_{1}$, then dividing $a_{2}$ by the appropriate row-sum of $A_{2}$, and then multiplying the two together.

The last lemma is a piece of "folklore" from linear algebra.

Lemma 3. Let $A$ be an $m \times n$ matrix and let $B$ be an $n \times m$ matrix, with entries in some field. If $p_{A B}(\lambda)$ and $p_{B A}(\lambda)$ are the characteristic polynomials of $A B$ and $B A$ respectively, then $\lambda^{m} p_{A B}(\lambda)=\lambda^{n} p_{B A}(\lambda)$.

Sketch of proof. See Exercise 3 of VII.5 of [7], or note that $\operatorname{tr}\left[(A B)^{k}\right]=\operatorname{tr}\left[(B A)^{k}\right]$ for all positive $k$ and apply Newton's identities.

\section{The MAIn ReSUlt}

Theorem 1. Let $G_{1}, G_{2}, \ldots, G_{n}$ be connected bipartite graphs with positive integer edge-weights and at least one edge each. Then $G_{1} \otimes G_{2} \otimes \cdots \otimes G_{n}$ has $2^{n-1}$ connected components, each of which is also a connected bipartite graph with positive integer edge-weights and at least one edge. The $\phi$-polynomials of the connected components are all equal up to a factor of a power of $\lambda$.

Proof. We shall give full details only for the case $n=2$; the general case follows exactly the same pattern, with no new ideas, but it is messy to notate. For $i=1,2$, let $\left(X_{i}, Y_{i}\right)$ be a bipartition of $G_{i}$. Let

$$
Z_{1}=\left(X_{1} \times X_{2}\right) \cup\left(Y_{1} \times Y_{2}\right) \quad \text { and } \quad Z_{2}=\left(X_{1} \times Y_{2}\right) \cup\left(Y_{1} \times X_{2}\right) .
$$

Let $H_{1}$ and $H_{2}$ be the (weighted) subgraphs of $G_{1} \otimes G_{2}$ induced by $Z_{1}$ and $Z_{2}$ respectively. From the definition of tensor product we see that the edge-weights of a 
tensor product are the product weights, and that the only edges of $G_{1} \otimes G_{2}$ are those between the two parts of $H_{1}$ and between the two parts of $H_{2}$. It is straightforward to verify that the connectedness of $G_{1}$ and $G_{2}$ implies the connectedness of $H_{1}$ and $H_{2}$, proving the first part of the theorem.

If the adjacency matrix of $G_{i}$ is

$$
\left(\begin{array}{cc}
0 & A_{i} \\
A_{i}^{T} & 0
\end{array}\right)
$$

then the adjacency matrices of $H_{1}$ and $H_{2}$ are

$$
\left(\begin{array}{cc}
0 & A_{1} \otimes A_{2} \\
A_{1}^{T} \otimes A_{2}^{T} & 0
\end{array}\right) \quad \text { and } \quad\left(\begin{array}{cc}
0 & A_{1} \otimes A_{2}^{T} \\
A_{1}^{T} \otimes A_{2} & 0
\end{array}\right) .
$$

Depending on whether $\left|X_{1}\right|\left|X_{2}\right| \leq\left|Y_{1}\right|\left|Y_{2}\right|$ or $\left|X_{1}\right|\left|X_{2}\right|>\left|Y_{1}\right|\left|Y_{2}\right|, \phi_{H_{1}}(\lambda)$ is the characteristic polynomial of either

$$
S\left(A_{1} \otimes A_{2}\right) S\left(A_{1}^{T} \otimes A_{2}^{T}\right) \quad \text { or } \quad S\left(A_{1}^{T} \otimes A_{2}^{T}\right) S\left(A_{1} \otimes A_{2}\right) .
$$

By Lemma 3 (taking the field to be $\mathbb{Q}$, say), these two characteristic polynomials differ only by a factor of a power of $\lambda$, and we are concerned only with equality up to such a factor, so it suffices to consider just the first of the two cases. Now

$$
\begin{aligned}
S\left(A_{1} \otimes A_{2}\right) S\left(A_{1}^{T} \otimes A_{2}^{T}\right) & =\left[S\left(A_{1}\right) \otimes S\left(A_{2}\right)\right]\left[S\left(A_{1}^{T}\right) \otimes S\left(A_{2}^{T}\right)\right] \\
& =\left[S\left(A_{1}\right) S\left(A_{1}^{T}\right)\right] \otimes\left[S\left(A_{2}\right) S\left(A_{2}^{T}\right)\right],
\end{aligned}
$$

where the first equality follows from Lemma 2 and the second equality may be justified by noting that the dimensions of the matrices $A_{1}$ and $A_{1}^{T}$ and of the matrices $A_{2}$ and $A_{2}^{T}$ are compatible for multiplication, so that the matrices may be interpreted as linear transformations, when the equality is obvious. Now a similar argument shows that $\phi_{H_{2}}(\lambda)$ is, up to a factor of a power of $\lambda$, the characteristic polynomial of

$$
\left[S\left(A_{1}\right) S\left(A_{1}^{T}\right)\right] \otimes\left[S\left(A_{2}^{T}\right) S\left(A_{2}\right)\right] .
$$

This differs from what we have for $H_{1}$ only by the interchange of $S\left(A_{2}\right)$ and $S\left(A_{2}^{T}\right)$. But by Lemma $3, S\left(A_{2}\right) S\left(A_{2}^{T}\right)$ and $S\left(A_{2}^{T}\right) S\left(A_{2}\right)$ have the same spectra up to the multiplicity of zero. The proof is now concluded by noting that the spectrum of a tensor product of two square matrices is obtained by taking the product of an eigenvalue of one matrix with an eigenvalue of the other in all possible ways, counting multiplicities ([2], Theorem 2.23).

Corollary 1. Let $G_{1}=\left(X_{1}, Y_{1}\right)$ and $G_{2}=\left(X_{2}, Y_{2}\right)$ be complete bipartite graphs with edges weighted by independent indeterminates and with at least one edge each. Let $H_{1}$ and $H_{2}$ be the two connected components of $G_{1} \otimes G_{2}$ with vertex sets

$$
Z_{1}=\left(X_{1} \times X_{2}\right) \cup\left(Y_{1} \times Y_{2}\right) \quad \text { and } \quad Z_{2}=\left(X_{1} \times Y_{2}\right) \cup\left(Y_{1} \times X_{2}\right)
$$

respectively. Then

$$
\frac{t\left(H_{1}\right)}{t\left(H_{2}\right)}=\left(\frac{\prod_{v \in X_{1}} \operatorname{deg}(v)}{\prod_{v \in Y_{1}} \operatorname{deg}(v)}\right)^{\left|X_{2}\right|-\left|Y_{2}\right|}\left(\frac{\prod_{v \in X_{2}} \operatorname{deg}(v)}{\prod_{v \in Y_{2}} \operatorname{deg}(v)}\right)^{\left|X_{1}\right|-\left|Y_{1}\right|}
$$


Proof. We will prove the result with arbitrary positive integer values in place of the indeterminates. This suffices because the given equation is a polynomial identity (after denominators are cleared).

The bijection between the edges of $H_{1}$ and $H_{2}$ that sends $\left(x_{1} \times x_{2}, y_{1} \times y_{2}\right)$ to $\left(x_{1} \times y_{2}, y_{1} \times x_{2}\right)$ is weight-preserving, so that the sums of the weights of the edges of $H_{1}$ and $H_{2}$ are equal. So by Lemma 1 we have

$$
t\left(H_{1}\right)\left(\prod_{v \in Z_{2}} \operatorname{deg}(v)\right)\left(\prod_{i=2}^{k_{2}}\left(1-\lambda_{i}^{\prime}\right)\right)=t\left(H_{2}\right)\left(\prod_{v \in Z_{1}} \operatorname{deg}(v)\right)\left(\prod_{i=2}^{k_{1}}\left(1-\lambda_{i}\right)\right),
$$

where the $\lambda_{i}$ are the roots of $\phi_{H_{1}}$ and the $\lambda_{i}^{\prime}$ are the roots of $\phi_{H_{2}}$. By Theorem 1, the $\lambda_{i}$ and the $\lambda_{i}^{\prime}$ are the same except that possibly there are more zeroes in one case. But zero roots simply contribute a factor of one to the $i$-products in the above equation and hence they may be ignored. Furthermore, these products are nonzero since the complexity of a complete bipartite graph is nonzero, so we may cancel them on both sides, leaving

$$
t\left(H_{1}\right) \prod_{v \in Z_{2}} \operatorname{deg}(v)=t\left(H_{2}\right) \prod_{v \in Z_{1}} \operatorname{deg}(v) .
$$

An examination of the adjacency matrices as in the proof of Theorem 1 shows that the product of the degrees of the vertices of $H_{1}$ is

$$
\left(\prod_{v \in X_{1}} \operatorname{deg}(v)\right)^{\left|X_{2}\right|}\left(\prod_{v \in X_{2}} \operatorname{deg}(v)\right)^{\left|X_{1}\right|}\left(\prod_{v \in Y_{1}} \operatorname{deg}(v)\right)^{\left|Y_{2}\right|}\left(\prod_{v \in Y_{2}} \operatorname{deg}(v)\right)^{\left|Y_{1}\right|}
$$

and the product of the degrees of the vertices of $\mathrm{H}_{2}$ is

$$
\left(\prod_{v \in X_{1}} \operatorname{deg}(v)\right)^{\left|Y_{2}\right|}\left(\prod_{v \in Y_{2}} \operatorname{deg}(v)\right)^{\left|X_{1}\right|}\left(\prod_{v \in Y_{1}} \operatorname{deg}(v)\right)^{\left|X_{2}\right|}\left(\prod_{v \in X_{2}} \operatorname{deg}(v)\right)^{\left|Y_{1}\right|} .
$$

Substituting these expressions into the equation yields the desired result.

We remark that this corollary subsumes all previously known special cases$P_{2 n+1} \otimes P_{2 n+1}$ (Stanley's conjecture), $P_{2 n+1} \otimes P_{2 m+1}$ with $m \neq n$ (Knuth and Ciucu), $P_{2 n+1} \otimes P_{2 m+1}$ where one of the paths has arbitrary weights on its edges (Ciucu), and $K_{m, n} \otimes P_{2 r+1}$ (Sagan [12]), where $K_{m, n}$ is a complete bipartite graph. For example, to recover Stanley's conjecture, let $X_{1}$ and $Y_{2}$ have $n$ vertices each and let $X_{2}$ and $Y_{1}$ have $n+1$ vertices each. Assign values of zero or one to the indeterminate weights appropriately so that $G_{1}$ and $G_{2}$ reduce to graphs equivalent to $P_{2 n+1}$. The product of the degrees of one part of $P_{2 n+1}$ divided by the product of the degrees of the other part equals two. We obtain a ratio of $2^{1} \times\left(\frac{1}{2}\right)^{-1}=4$ from Corollary 1, explaining the factor of four in Stanley's conjecture.

\section{Concluding Remarks}

1. We mentioned in the introduction that the $Q$-spectrum of any graph behaves well under tensor product, but we did not show this fact explicitly in our proof since it was not needed. Here are the details. If $G$ is a graph without isolated vertices and with adjacency matrix $A$, then the $Q$-polynomial of $G$ is defined by

$$
Q_{G}(\lambda) \stackrel{\text { def }}{=} \operatorname{det}[\lambda I-S(A)] .
$$


Thus, from Lemma 2,

$$
Q_{G_{1} \otimes G_{2}}(\lambda)=\operatorname{det}\left[\lambda I-S\left(A_{1} \otimes A_{2}\right)\right]=\operatorname{det}\left[\lambda I-S\left(A_{1}\right) \otimes S\left(A_{2}\right)\right] .
$$

But as we have already remarked, the eigenvalues of a tensor product are just the pairwise products of the individual eigenvalues ([2], Theorem 2.23). So the $Q$-spectrum of $G_{1} \otimes G_{2}$ is easily computed from the $Q$-spectra of $G_{1}$ and $G_{2}$.

2. It is natural to ask if the $Q$-spectrum method can be applied to other graph constructions, such as the sum or more generally any NEPS ([2], §2.5). Unfortunately, the $Q$-spectrum appears to behave badly with respect to the sum. Curiously, the Laplacian spectrum behaves well with respect to sum (see [9]) but badly with respect to tensor product. Perhaps some amalgamation of the two, such as one of the multivariate spectra suggested in [2], §1.3, will solve the problem of enumerating spanning trees in any NEPS, but I have not been able to do so.

3. Igor Pak [10] has suggested that perhaps the planarity assumption in Knuth's proof might be weakened to a matroid duality assumption. However, at present there is no matroid generalization of Stanley's conjecture known.

4. It is tempting, in light of how simple and striking the statement of Corollary 1 is, to look for an insightful combinatorial proof. Sagan [12] has obtained an explicit formula for the complexities of the connected components of $K_{m, n} \otimes P_{2 r+1}$; for one component it is

$$
(2 m)^{r(n-1)} m^{r-1}(2 n)^{m(r-1)} n^{2 m-1} .
$$

He has suggested that this formula and/or Corollary 1 might be proved using Prüfer codes, along the lines of [6]. The best I have done with Sagan's suggestion is to prove the special case of Corollary 1 when there exists a weighting of the vertices of $G_{1}$ and $G_{2}$ such that the weight of each edge is the product of the weights of its endvertices. In this case, a Prüfer code argument gives an explicit factorization of $t\left(H_{1}\right)$ and $t\left(H_{2}\right)$ into linear functions of the vertex weights. From this Corollary 1 follows easily.

5. By relabelling if necessary we may assume in Corollary 1 that $\left|X_{1}\right| \geq\left|Y_{1}\right|$ and $\left|X_{2}\right| \geq\left|Y_{2}\right|$, so that what looks like the denominator really is the denominator. It is easy to see that the fraction on the right-hand side is in lowest terms, so by unique factorization it follows that the polynomial $t\left(H_{1}\right)$ is divisible by the numerator of the right-hand side (and of course $t\left(H_{2}\right)$ is divisible by the denominator). One can then ask for a combinatorial interpretation of the quotient. In the case where $\left|X_{1}\right|=\left|Y_{1}\right|$ and $G_{2}$ is a path with three vertices I have a combinatorial proof that the quotient enumerates the spanning trees of $G_{1}$, but the argument seems difficult to generalize. If $G_{2}$ is an arbitrarily long path, I can show that the quotient has nonnegative coefficients but it does not seem to enumerate anything simple.

\section{ACKNOWLEDGMENTS}

Perhaps the greatest breakthrough in proving the main result of this paper came when I realized that it had something to do with the fact that $(A \otimes B)\left(A^{T} \otimes B^{T}\right)$ and $\left(A \otimes B^{T}\right)\left(A^{T} \otimes B\right)$ have essentially the same characteristic polynomials. I am grateful to John Stembridge for showing me how to prove this fact. I also wish to thank Mihai Ciucu for suggesting that Theorem 1 could be stated for arbitrary $n$ and not just $n=2$. Finally, thanks to Richard Stanley for introducing me to this problem and to Bruce Sagan, Mihai Ciucu, Igor Pak, and Jim Propp for expressing 
their enthusiasm about Corollary 1 during a period when I was doubting that it was interesting.

\section{REFERENCES}

1. M. Ciucu, Perfect matchings, spanning trees, plane partitions and statistical physics, Ph.D. thesis, University of Michigan, Ann Arbor, MI, 1996.

2. D. Cvetković, M. Doob, and H. Sachs, Spectra of Graphs: Theory and Application, Academic Press, New York, NY, 1980. MR 81i:05054

3. D. Cvetković and I. Gutman, A new spectral method for determining the number of spanning trees, Publ. Inst. Math. (Beograd) 29 (1981), 49-52. MR 83f:05046

4. N. Elkies, G. Kuperberg, M. Larsen, and J. Propp, Alternating-sign matrices and domino tilings, J. Algebraic Combin. 1 (1992), 111-132 and 219-234. MR 94f:52035; MR 94f:52036

5. M. Farzan and D. A. Waller, Kronecker products and local joins of graphs, Can. J. Math. 29 (1977), 255-269. MR 55:2625

6. N. Hartsfield and J. S. Werth, Spanning trees of the complete bipartite graph, Topics in Combinatorics and Graph Theory (R. Bodendiek and R. Henn, eds.), Physica-Verlag, Heidelberg, 1990. MR 91m:05100

7. T. Hungerford, Algebra, Holt, Rinehart and Winston, Inc., New York, NY, 1974. MR 50:6693

8. D. Knuth, Aztec diamonds, checkerboard graphs, and spanning trees, preprint.

9. G. Kreweras, Complexité et circuits eulériens dans les sommes tensorielles de graphes, J. Combin. Theory B24 (1978), 202-212. MR 80c:05099

10. I. Pak, personal communication.

11. H. Sachs, On the number of spanning trees, Proc. 5th Brit. Combin. Conf., Aberdeen 1975 (C. St. J. A. Nash-Williams and J. Sheehan, eds.), Winnipeg, 1976, pp. 529-535. MR 55:167

12. B. Sagan, personal communication.

13. R. Stanley, Research problem 251: Spanning trees of Aztec diamonds, Discrete Math. 157 (1996), 383-385.

14. H. N. V. Temperley, Combinatorics, London Math. Soc. Lecture Notes Series, vol. 13, 1974, pp. 202-204. MR 82i:05001

Department of Mathematics, University of Michigan, Ann Arbor, Michigan 48109

E-mail address: tchow@umich.edu 